\title{
TRANSPUTER-BASED PARALLEL SYSTEMS FOR PERFORMANCE EVALUATION OF BIDIRECTIONAL ASSOCIATIVE MEMORY
}

\author{
Indu Mahadevan \\ Dept. of Computer Science and Automation, \\ L.M. Patnaik \\ Microprocessor Applications Laboratory, \\ Supercomputer Education and Research Centre and \\ Dept. of Computer Science and Automation, \\ Indian Institute of Science, \\ Bangalore 560012. \\ E-MAIL : lalit@vigyan.ernet.in
}

\begin{abstract}
In this paper, we discuss parallel implementation of an artificial neural network for pattern association, the Bidirectional Associative Memory (BAM). Transputer-based parallel architecture8like hypercube, mesh and linear array are used to exploit the parallelism in BAM. A comparitive study of utilization and speedup for various architectures is made. Hypercube performs better in terms of utilization and speedup when compared to the other architectures.
\end{abstract}

\section{INTRODUCTION}

Bidirectional Associative Memory (BAM) is a model of an artificial neural network useful in areas of pattern recognition in a noisy environment. The BAM is a two layered network that can map any two binary patterns[3][4], which are stored in the encoding stage and recalled in the decoding stage.

The BAM operations are compute-intensive in nature and mainly involve vector matrix multiplications suitable for parallel implementation resulting in faster training and recalling operations. Since not much attention has been focussed on the architectural aspects of BAM so far, this paper attempts to exploit the parallelism inherent in BAM. The main motivation behind this work has been to implement BAM on the various parallel architectures like hypercube, mesh and linear array using transputers and find the speedup and utilization factors and hence arrive at the most suitable architecture for effective parallel implementation of BAM.

The paper is organised as follows. Section 2 gives an outline of BAM and highlights why transputers have been used for simulation. The parallelism in BAM has been discussed in section 3 . Section 4 deals with some simulation details. Simulation results are shown in section 5. Section 6 deals with the applications and results and conclusions are discussed in section 7 


\section{OUTLINE OF BAM AND TRANSPUTERS FOR SIMU- LATION OF BAM}

Paired data association $\left(A_{i}, B_{i}\right)$ are stored as an $\mathrm{n}$ times $\mathrm{p}$ matrix $\mathrm{M}$ where $A_{i}$ is a row vector of dimension $\mathrm{n}$ and $B_{i}$ is a row vector of dimension p. Matrix $\mathrm{M}$ is obtained as the sum of the outer products of the data pairs,

$M=A_{i}^{T} B_{i}$.

Recalling in the forward direction of any pattern $A_{i}$ is obtained as shown below.

$A_{i} M=X i$.

Recalling in the backward direction of any pattern $B_{i}$ is obtained as shown below.

$B_{i} M^{T}=X_{i}$.

Transputers have been used for the implementation of various parallel architectures for the simulation of BAM. Transputers and neural networks belong to the class of communicating sequential proces (CSP) and hence it is easy to model and test neural networks. Transputers do not face limitations of microprocessor systems like mutual exclusion and contention and the four links of transputers can be easily configured into various topologies. These advantages have prompted us to use transputers for this simulation study.

\section{PARALLELISM IN BAM}

Processors execute the same program on different pieces of data exploiting data parallelism[1]. The parallelism in the operations of training and recalling are discussed using the example of associating two pattern pairs $(\mathrm{A}, \mathrm{B})$,

$$
\begin{aligned}
& A=(a \perp \text { a } 2 \text { a3 a4 }) \\
& B=(\text { bl b2 b3 b4). }
\end{aligned}
$$

We will consider the BAM being implemented on two transputers (hereafter referred to as $\operatorname{Trl}$ and $\operatorname{Tr} 2$ ) and consider the speedup obtained in training and recalling the BAM. The following notations are used in the discussion :

$n A$ and $n B$ are the number of elements in row vectors $A$ and B respectively, tm and $t a$ are the time taken for multiplication and addition respectively. $\boldsymbol{T} \boldsymbol{s}$ represents the time taken by a serial processor for a particular operation and Tp is the time taken by the parallel version of the same program, $\boldsymbol{x}$ is the number of patterns stored,$N$ is the number of transputers and $[\boldsymbol{n}\rceil$ is the ceiling function. We define speedup as shown below,

Speedup $=T s /(T p+T c)$

where $\mathrm{Tc}$ is the communication time to bring in data from the host or for interprocessor communication.

To train, the matrix $M$ is obtained using the outer product $A^{T} B=\mathrm{M}$. Each element in the pattern corresponds to a neuron in a layer. In our example, we have considered four neurons in each of the two layers. The elements $\boldsymbol{o}$ pattern $A$ and $B$ can be divided such that elements $a 1$ and a2 are stored in $\operatorname{Trl}$ and $\mathrm{a} 3$ and $a 4$ are stored in $\operatorname{Tr} 2$ and Vector $B$ is stored in both the transputers. Trl calculates rows 1 and 2 of the resultant matrix $\mathbf{M}$ while rows $\mathbf{3}$ and $\mathbf{4}$ can be calculated in $\mathbf{T} \mathbf{2}$ simultaneously.

We have,

$T s=x \times(n A \times n B) \times(t m+t a)$

$T p=\left(x x\left\lceil\frac{n A}{N}\right\rceil x n B\right) x(t m+t a)$

During forward recall of a pattern $\mathrm{X}$, the following operation must be carried out,

$$
X M=Y \text {. }
$$

The elements of vector $X, z 1$ and 22 are stored in Trl and $x 3$ and $x 4$ are stored in $\operatorname{Tr} 2$ since the first two rows of $M$ are in $\operatorname{Trl}$ and the other two in $\operatorname{Tr} 2$. $\operatorname{Tr} 1$ calculates the product of $x 1$ and elements of row $\mathbf{1}$ of $\mathbf{M}$ and product of 22 and elements of row 2 of $\boldsymbol{M}$. Simultaneously,Tr2 calculates the product of $\boldsymbol{x} \mathbf{3}$ and elements of row $\mathbf{3}$ of $\mathbf{M}$ and product of $\boldsymbol{x} 4$ and elements of row $\mathbf{4}$ of $\boldsymbol{M}$. After this process, 
the partial results belonging to the elements of $\boldsymbol{Y}$ matrix available in the two transputers are shifted and added to obtain the final result.

We have, $\mathbf{T s}=(n A \times n B \times t m)+((n A-1) \times n B \times$ ta $)$

$T p=\left(\left\lceil\frac{n A}{N}\right\rceil \times n B \times t m\right)+\left(\left(\left\lceil\frac{n A}{N}\right\rceil-1\right) \times n B \times t a\right)+((N-1) \times n B \times$ ta $\left.)\right)$

The last term in the above equation represents the extra number of additions required because of the method in which the data are divided among the transputers.

For backward recall of a pattern $\boldsymbol{Y}$,we must perform the following operation,

$$
Y M^{T}=X \text {. }
$$

The first two columns of matrix $M^{T}$ are in $\operatorname{Trl}$ and the other two columns are in $\operatorname{Tr} 2$. So the entire $\boldsymbol{Y}$ vector is sent to $\operatorname{Tr} 1$ and $\operatorname{Tr} 2$. While $\operatorname{Tr} 1$ calculates $x 1$ and 22 , $\operatorname{Tr} 2$ can simultaneously obtain 23 and 24.

We have,

$T s=(n A \times n B \times t m)+(n A \times(n B-1) \times t a)$

$T p=\left(\left\lceil\frac{n A}{N}\right\rceil \times n B \times t m\right)+\left(\left\lceil\frac{n A}{N}\right\rceil \times(n B-1) \times t a\right)$

\section{SOME SIMULATION DETAILS}

Various features of transputers such as the ability for communicating parallely on their four links have been exploited to make the best use of the diameter of a topology and send data in parallel. The Occam language has the construct PAR for parallel execution of many processes.

The simulation was carried out on two IMS B003 boards each having four T800 transputers configured as a square. The remaining links have been used to configure the other topologies. The host is a T800 transputer.

A general outline of the simulator is given below. The SEQ and PAR constructs of Occam imply sequential and parallel operation of the processes which are shown below with an indentation of two spaces below them.

\subsection{Pseudocode of program as a whole}

\section{SEQ}

... Get number of neurons and number of transputers

available

... Divide neurons among various transputers

PAR in all transputers do

... For all neurons in the transputer perform the specified operations of training or recalling

\subsection{Pseudocode for operations in any particular transputer}

SEQ

... Receive input data from a zeighbouring transputer PAR

... Send input data to all other neighbours PAR

... Perform the operation specified

... Receive output data from all the neighbours

... Send output data to other neighbours or the host transputer 


\section{SIMULATION RESULTS}

The BAM has been simulated on a single transputer and on multitransputers with the various topologies namely hypercube, mesh and linear array. Since this network is being used for numeral recognition, the patterns being associated are numerals and the layers of the BAM have equal number of neurons. The simulation is carried out for two situations as shown below.

1. The number of transputers is maintained a constant and the number of neurons in each layer is varied. Results of speedup are shown in figures 1,2 and 3 .

2. The number of neurons in both the layers is maintained constant and the number of transputers is varied. Results of speedup are shown in figures 4,5 and 6 .

Two cases are considered in both situations mentioned above. In the first case, the setup time, the time to load the initial data from the host transputer to the other transputers, is also taken into account. In the second case, the setup time is not considered.

Utilization of the system is defined as the average of the utilization of all the transputers in the system. For each transputer, utilization is defined as the ratio of the time spent in computing the results to the total time taken for the entire training or recalling operation. If Tcalc and Tcomm are the computation and communication times of any operation, utilization is defined as given below.

Utilization $=T$ calc $/(T$ calc + Tcomm $)=T c a l c / T$

(of each transputer)

If the total number of transputers used is $\mathrm{N}$, utilization of the system is given by $U=\sum_{i=1}^{N} T$ calc $_{i} / \mathrm{NT}$

Figs 7,8 and 9 show the utilization of the system for various topologies.

\section{APPLICATIONS}

This implementation is being used for numeral recognition purposes. The autoassociative form of learning has been used wherein, a mapping is created from an input pattern to itself. Handwritten characters written on a grid are obtained from several sources and are used as the input for recognition. In our application, we have used eighty neurons in each layer to represent the numerals. At present, we can store any four numerals. About twenty bib of the eighty bit patterns were changed randomly and the pattern was successfully recalled seventy percent of the time.We hope to extend the capacity of BAM to store and recall more aumerads correctly using multiple training[5].

\section{RESULTS AND CONCLUSIONS}

Hypercube topology gives a better speedup when compared to mesh and $1 \mathrm{i}$ a $\mathrm{r}$ array during training and recalling in the BAM. (Figs 1,2 and 3). This could be explained as follows. In all the topologies considered, the problem can be mapped in such a way that the computation time taken is the same. But during computation, there is a need for communication between the processors. In the case of a hypercube topology, each processor at a node has more neighbouring processors than other toplogies. Hence, in mapping the problem onto a topology we can make the best use of diameter of the topology to reduce the communication time between processors. Since the hypercube topology has a smaller diameter when compared to mesh and linear array, it is faster during training and recalling patterns in the BAM. When the setup times are not considered(iadicated by dotted lines in the figures), the speedup obtained is better and all topologies perform equally well during the training process since there is not much communication involved between processors. From Figs 1,2 and 3 we find that the speedup obtained when the number of transputers is increased is not linear. The two transputer versions of the different topologies yield a speedup close to the maximum speedup of two. But as the number of transputers is increased to eight, we find that the speedup is far below the theoretical maximum speedup of eight since more communication is involved which takes more time when compared to the time taken for computation. From Figs 4, 5 and 6 we find that when the setup time is not considered, speedup 
obtained during training is much closer to the maximum value of eight when comnared to the recalling operations, due to the limited communication between the processors during training operation.

Utilization of the system drops as the number of transputers increases because communcation time starts increasing with the computation time remaining the same. In the case of a four transputer network, we find that the mesh(or 2D hypercube) is better utilized than the linear array because the system spends less time in communication during a training or recalling operation when compared to the linear array. For the same reason, the eight transputer hypercube performs better than the mesh and linear array. When the setup time is not considered, we find that all the topologies are utilized equally well during training.

In problems of the nature of $B A M$, mainly involving vector matrix multiplications, the computations can be divided almost equally among all the transputers. So most topologies would perform equally well. The diameter of the topology can decide the communication time and hence the overall performance. We plan to extend our work to other topologies like a tree for better speedup and utilization factors.

\section{REFERENCES}

[1] David Suter and Xiaolan Deng, Neural liet Simulation on Transputers, IEEE Computer, 1988, pp 894 - 697.

[2] P. Koikkalainer and E. Oja, Specification and Implementation Environment for Neural Networks using Communicating Sequential Processes, IEEE ICNN, 1987, pp 1-533-1-540.

[3] B.Kosko, Adaptive Bidirectional Associative Memories. Applied Optics, Vol 26, December 1987, pp 4947 - 4960.

[4] B. Kosko, Bidirectional Associative Memories, IEEE Trans. on Systems, $M a n$ and Cybernetics, Vol.18, Febrauary 1988, pp 49 - 59 .

[5] Yeou-Fang Wang, J.B.Cruz and J.R.Mulligan, An Enhanced Bidirectional Associative Memory, IEEE INNS ICNN, 1989, pp 1-105-1-110.
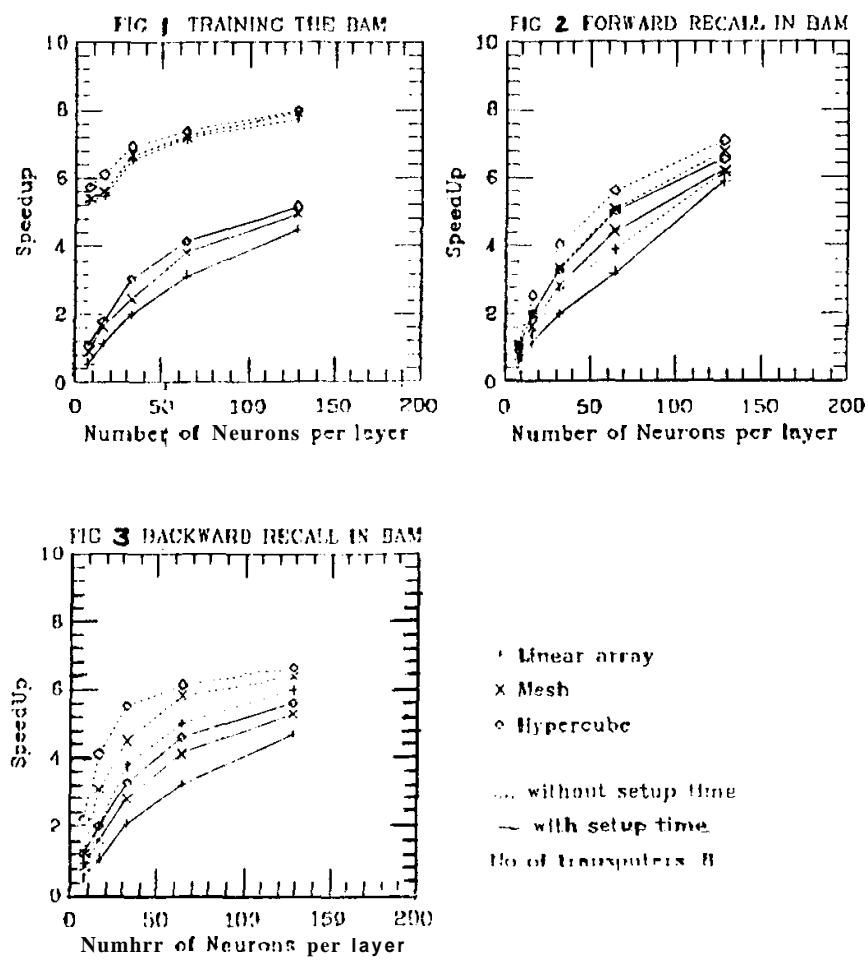

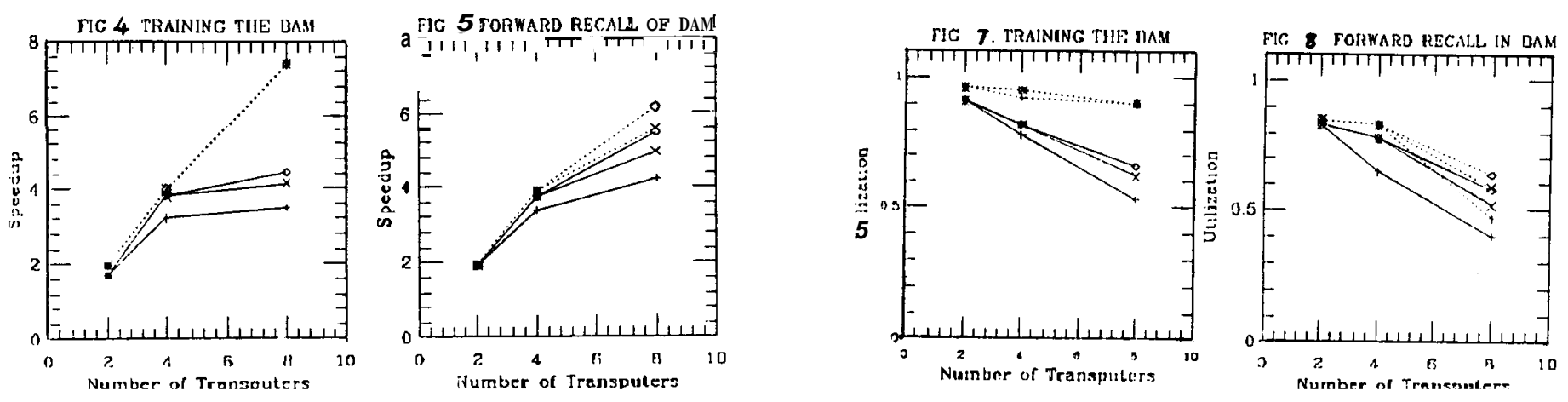

莣

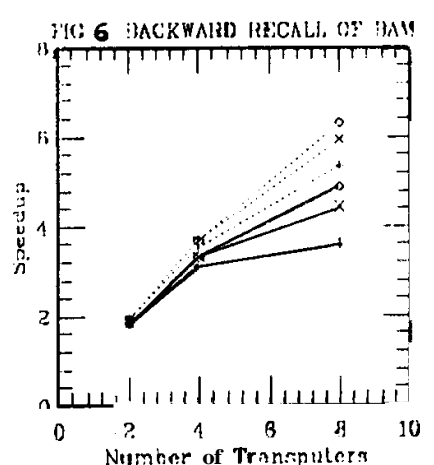

+ Iencar orras

$X$ Mesh

- Hypercube

.... without setup tine

-- with setup time

Neurons per layer:b0
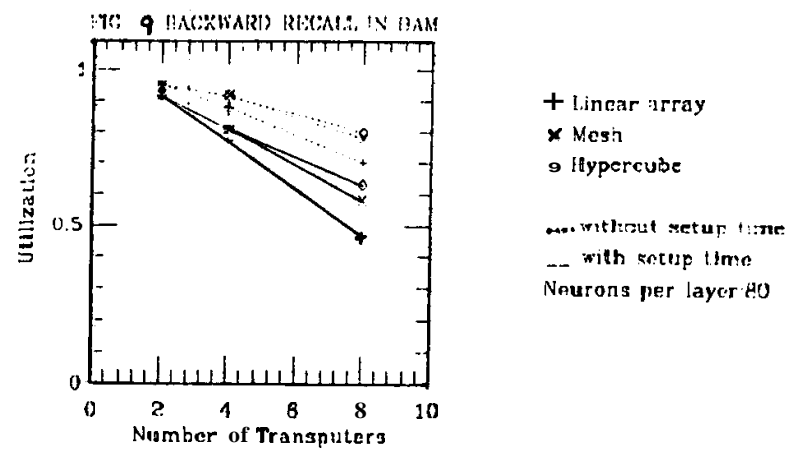